\title{
Identification and sequence analysis of prolactin receptor and its differential expression profile at various developmental stages in striped hamsters
}

\author{
Huiliang $\mathrm{Xue}^{1 \oplus}$, Jinhui $\mathrm{Xu}^{1 \oplus}$, Ming $\mathrm{Wu}^{1 \oplus}$, Lei $\mathrm{Chen}^{1 \oplus}$, and Laixiang $\mathrm{Xu}^{1 \oplus}$ \\ ${ }^{1}$ College of Life Sciences, Qufu Normal University, Qufu, Shandong, China
}

\begin{abstract}
Prolactin (PRL) plays critical roles in regulation of biological functions with the binding of specific prolactin receptor (PRLR). Revealing the expression patterns of PRLR at different developmental stages is beneficial to better understand the role of PRL and its mechanism of action in striped hamsters. In this study, the cDNA sequence of PRLR (2866-base-pairs) was harvested from the pituitary of mature female striped hamsters (Cricetulus barabensis) that contains an 834-base-pair $5^{\prime}$-untranslated region (1-834 bp), a 1848-base-pair open reading frame (835-2682 bp), and a 184-base-pair 3'-untranslated region (26832866). The 1848-base-pair open reading frame encodes a mature prolactin-binding protein of 592 amino acids. In the mature PRLR, two prolactin-binding motifs, 12 cysteines, and five potential Asn-linked glycosylation sites were detected. Our results showed that the PRLR mRNA quantity in the hypothalamus, pituitary, ovaries, or testis was developmental-stage-dependent, with the highest level at sub-adult stage and the lowest level at old stage. We also found that PRLR mRNAs were highest in pituitary, medium level in hypothalamus, and lowest in ovaries or testis. PRLR mRNAs were significantly higher in males than in females, except in the hypothalamus and pituitary from 7-week-old striped hamsters. Moreover, the PRLR mRNAs in the hypothalamus, pituitary, and ovaries or testis were positively correlated with the expression levels of GnRH in the hypothalamus. These results indicated that the PRLR has conserved domain in striped hamster, but also possesses specific character. PRLR has multiple biological functions including positively regulating reproduction in the striped hamster.
\end{abstract}

Key words: PRLR; Striped hamster; Developmental stages

\section{Introduction}

The striped hamster (Cricetulus barabensis) is one of main rodent pests in northern China farmland that has high reproductive capacity. It generally reproduces three times a year, and the litter size is of four to nine for every parity (1). The high reproductive activity is one of main reasons leading to the population fluctuation (2). In general, rodents play an important role in maintaining the ecological balance, however, when there is an outbreak, rodents cause enormous damage to agricultural production (3).

Prolactin ( $P R L)$ is a single-chain peptide hormone that is mainly synthesized and secreted by prolactin cells in the anterior pituitary, and acts on breast development, lactation maintenance, reproduction (4), as well as on growth and development, metabolism, immuno-regulation, energy balance, and behavior $(5,6)$. PRL acts by binding to a specific membrane prolactin receptor (PRLR) $(7,8)$, which belongs to the class I cytokine receptor superfamily (9). PRLR is a single membrane-bound protein expressed in the hypothalamus, pituitary, breast, ovary, testis, liver, and many other tissues of mammalians (10). The circulating levels of PRL are highly correlated with the levels of PRLR mRNA in both the pituitary gland and hypothalamus, which indicates that PRL positively regulates the PRLR expression in the hypothalamus (11) and biological actions of PRL are mediated by PRLR simultaneously.

The expression levels of PRLR are much higher in the hypothalamus and pituitary gland than in other tissues (12). Therefore, PRLR participates in the biological function of PRL, and its specific function and expression level are tissue-dependent. However, the mechanism involved in PRL binding to PRLR with the transferring of hormonal signal inside the cell remains unknown. The study on primary structure and expression patterns of PRLR may facilitate the understanding of the PRL mechanism of action. Since the action of PRL is initiated by its specific receptor on the cell surface (13), a detailed characterization of PRLR is necessary to further understand the PRL 
action mechanism. The cysteine residues and $\mathrm{N}$-linked glycosylation sites are important to build specific constructs and execute specific activities for PRLR. Whether conserved cysteine residues and $\mathrm{N}$-linked glycosylation sites also exist in the striped hamster PRLR is still not well understood.

In humans, prolactin is secreted daily with the highest level during sleep and the lowest level during waking hours $(14,15)$, which may be regulated by the suprachiasmatic nuclei of the hypothalamus (16). The transcription of PRLR in the ovaries is a complicated process regulated by developmental stage and hormones (17). The expression of PRLR in the hypothalamus is increased by prolactin in serum (18). Estrogens can also increase the expression level of PRLR in the brain (19). Therefore, the PRLR expression profile may vary with the different developmental stages.

To date, the PRLR sequence character and the PRLR mRNA changes in various developmental stages in the striped hamster are little understood. Therefore, our present study used the mature female striped hamster as an experimental model to reveal the cDNA sequence and the deduced amino acid sequence of PRLR and examine the expression patterns of PRLR at different developmental stages in striped hamsters.

\section{Material and Methods}

\section{Preparation of animals and tissues}

The striped hamsters (Cricetulus barabensis) used in this study were captured by live-trap method using iron cages in the fields of Puwang Village, Yinan County, Linyi City of Shandong Province, China. Six mature female striped hamsters were used to make cDNA libraries. Eighteen male and female animals (six hamsters of 7 weeks $(18 \pm 1 \mathrm{~g}), 13$ weeks $(24 \pm 1 \mathrm{~g})$, and 1.5 years old $(30 \pm 1 \mathrm{~g}))$ were used to examine the PRLR expression profile. The weight among animals in the same developmental stage and same sex deviated no more than $1 \mathrm{gram}$. Animals were treated in accordance with the Animal Ethics Committee of Qufu Normal University.

The selected animals were maintained on a 12-h light/ dark cycle schedule (lights on at 0400 hour) at Qufu Normal University (China) in the Animal Experiment Center at $20^{\circ} \mathrm{C}$ conditions for one week. The 7-week and 1.5-year old animals were then killed by $\mathrm{CO}_{2}$ asphyxiation, and were dissected to collect the hypothalamus, pituitary, and ovaries or testes, which were stored at $-80^{\circ} \mathrm{C}$. For the 13 -week hamsters, from 5 to $6 \mathrm{pm}$ after one week, the estrous cycle of females was examined by vaginal smears and the estrus period of males was judged by estrous behavior. When the animals were in estrous period, they were immediately killed by $\mathrm{CO}_{2}$ asphyxiation, and dissected to collect the hypothalamus, pituitary, and ovaries or testes tissues, which were stored at $-80^{\circ} \mathrm{C}$. All the experiments were approved by the Animal Ethics Committee of Qufu Normal University.

\section{Total RNA extraction}

Total RNA of the hypothalamus, pituitary, and ovaries or testis of the selected animals were extracted using RNA extraction reagent (TaKaRa, Japan). The purity and the concentration of total RNA were assessed by the ratio of D260 to D280 using an ultraviolet spectrophotometer (Eppendorf, Germany). The integrity of total RNA was also examined by agarose gel electrophoresis (AGE). Subsequently, all high-quality total RNA from the sampled tissues were reverse transcribed to cDNA using reverse transcriptase XL AMV (TaKaRa) with an Oligo deoxy thymidylate primer and random primers. All synthesized cDNA was stored at $-20^{\circ} \mathrm{C}$

\section{Preparation and screening of cDNA libraries}

The oligo (dT) and random primers were used to construct cDNA libraries from the pituitary glands of the six mature females. A sense primer (P1, 5'-GTCCAGACT CGCTGCAAGCC-3') and an antisense primer (P2, 5'-AG ATGCAGGTCATCATGCTA-3') were designed based on the conserved sequences of the golden hamster (XM_ 021231782.1), Mus musculus (NM_011169.5), and Rattus norvegicus (NM_001034111.1) PRLR and were used for the amplification of a cDNA fragment from the striped hamsters PRLR mRNA. A 193-bp fragment of PRLR cDNA amplified by RT-PCR using $\mathrm{P} 1$ and $\mathrm{P} 2$ primers was used as the probe to screen the cDNA libraries. Hybridization was performed in $20 \%$ formamide at $42^{\circ} \mathrm{C}$ and the filters were washed in $1 \times \mathrm{SSC}(0.15 \mathrm{M} \mathrm{NaCl}, 0.015 \mathrm{M}$ sodium citrate, $\mathrm{pH} 7$ ) at $42^{\circ} \mathrm{C}$.

\section{Gene characterization}

Similarity assessment and homology searches of the nucleotides and amino acids of the PRLR from the striped hamster were analyzed by NCBI BLAST (http://www.ncbi. nlm.nih.gov/blast) (20). SignalP 4.0 (http://www.cbs.dtu. dk/services/SignalP/) (21) and Tmpred (http://www.ch. embnet.org/software/TMPRED_form.html/) (21) were used to detect the protein signal peptide and the transmembrane structures, respectively.

\section{Real-time fluorescence quantitative polymerase chain reaction}

Based on the cloned nucleotide sequences of PRLR from the striped hamsters, primers of real-time quantitative polymerase chain reaction (RT-qPCR) of PRLR were designed using Beacon Designer 7.0 software (www.premierbiosoft.com). The primers of PRLR were F1 (5'- GCA TCTTTCCACCAGTTCC-3') and R1 (5'-TTGGCATCCT AAGGCAGT-3'). The primers of $\beta$-actin (as a reference gene) were F2 (5'-GAGACCTTCAACACCCCAGC-3') and R2 (5'-ATGTCACGCACGATTTCCC-3'). RT-qPCR was performed using an Agilent Stratagene Mx3000P detector (USA) with the Brilliant II SYBR Green qPCR Master mix (TaKaRa). Three repeats for each sample were performed during every RT-qPCR test. 
The volume of reaction system for RT-qPCR was $20 \mu \mathrm{L}$, including $10 \mu \mathrm{L}$ SYBR Green, $0.4 \mu \mathrm{L}$ forward primer and reverse primer $(10 \mu \mathrm{M})$, respectively, $2 \mu \mathrm{L}$ cDNA template, $0.3 \mu \mathrm{L}$ ROX, and $6.9 \mu \mathrm{L}$ DEPC $\mathrm{H}_{2} \mathrm{O}$. The reaction procedure was $10 \mathrm{~min}$ at $94^{\circ} \mathrm{C}$ for enzyme activation followed by 40 cycles of $1 \mathrm{~min}$ at $94^{\circ} \mathrm{C}, 1 \mathrm{~min}$ at $56^{\circ} \mathrm{C}$, and $1 \mathrm{~min}$ at $72^{\circ} \mathrm{C}$.

The fluorescence signal was collected during every PCR cycle at the renaturation step and was positively correlated with the quantity of the product. The specificity of the product was confirmed using melting curve analysis with a single peak per unique amplification, and the integrity of the product was examined by $2.5 \%$ AGE. The amplification efficiency for the specific primers was also tested using the standard curve, which should be from 90 to $110 \%$ (22). The expression level of PRLR mRNA is reported with the $2^{-\Delta \Delta C T}$ method (normalization to $\beta$-actin) (23).

\section{Statistical analysis}

The data are reported as means \pm SE. Statistical analysis was conducted using the independent sample $t$-test between two groups and the single-factor analysis of variance (ANOVA) among more than two groups. The differences were considered to be significant when $P<0.05$ and highly significant when $P<0.01$. Pearson's correlations were determined for PRLR relative expression levels in the hypothalamus with GnRH mRNA quantity in the hypothalamus. Data were analyzed using SPSS 17.0 (IBM, USA).

\section{Results}

\section{Character of the cDNA sequence and the deduced} amino acid sequence of PRLR

The cDNA sequence of PRLR (2866-base-pairs) in the pituitary of the mature females is shown in Figure 1, including an 834-base-pair 5'-untranslated region (1-834 bp), a 1848-base-pair open reading frame (835-2682 bp), and a 184-base-pair 3'-untranslated region (2683-2866). The 1848-base-pair open reading frame encoded a peptide of 615 amino acids, including a signal peptide of 23 amino acids and a mature prolactin-binding protein of 592 amino acids. The mature prolactin-binding protein contained the extracellular prolactin-binding region of 210 residues at amino-terminal, the transmembrane region of 24 residues, and the intracellular domain of 358 residues at carboxyl-terminal. In the mature prolactin-binding protein, 12 cysteines were detected, including 5 cysteines in the extracellular region, 1 cysteine in the transmembrane region, and 6 cysteines in the intracellular region. Five potential Asn-linked glycosylation sites were also detected, including 1 site in the signal peptide and extracellular region, respectively, and 3 sites in the intracellular domain. At the extracellular domain, two prolactin-binding motifs (WIKWS and WSRWG) were detected and two conserved parts (258-328 and 347-366 amino acids) were found in the intracellular domain.

\section{Expression profiles of PRLR among different developmental stages}

The expression levels of PRLR in the hypothalamus, pituitary, and ovaries or testis and that of $\mathrm{GnRH}$ in female or male animals were all developmental-stage-dependent. The highest expression levels of PRLR and GnRH were in 7-week-old animals, and the lowest in 1.5-year-old males and females. Medium expression levels of PRLR and $\mathrm{GnRH}$ were detected at 13-week estrous period. The expression levels of PRLR and $\mathrm{GnRH}$ from different developmental stages showed significant differences except for the difference of PRLR expression levels in the hypothalamus between the 7- and 13-week-old males (Figure 2).

\section{Expression profiles of PRLR among different tissues}

For 7-week-old females and males, expression of PRLR was significantly higher in pituitary and significant lower in ovaries or testis $(P<0.01)$. There was medium expression of PRLR in hypothalamus for the 7-week-old females and males (Figure $3 A$ and D). For 13-week-old estrous females and males, the expression levels of PRLR in hypothalamus and pituitary were significantly higher than in ovaries or testis $(P<0.01)$. Significantly higher expression of PRLR $(P<0.05)$ was found in pituitary than in hypothalamus in 13-week-old estrous males (Figure 3B and E). For 1.5-year-old females and males, the PRLR expression was significant higher in pituitary and hypothalamus than that in ovaries or testis (Figure $3 \mathrm{C}$ and $\mathrm{F}$ ). The results showed that the expression of PRLR was tissue-dependent in the striped hamsters.

\section{Expression profiles of PRLR between males and females}

For 7-week-old animals, no significant differences of PRLR mRNA were found in hypothalamus and pituitary between females and males (Figure $4 A$ and B). Significant differences of PRLR mRNA in hypothalamus were found between females and males at 13-week-estrous $(P<0.05)$ and 1.5-years $(P<0.01)$, respectively (Figure $4 A)$. Significantly higher PRLR mRNA was found in the pituitary of males than that of females at 13-week-estrous and 1.5years (Figure 4B). PRLR mRNA was significantly higher in male testis than that in female ovaries $(P<0.01)$ (Figure $4 \mathrm{C}$ ) and the GnRH mRNA was significant higher in male hypothalamus than that in female hypothalamus $(P<0.01)$ (Figure 4D) in 7- and 13-week-old, and 1.5year-old striped hamsters.

\section{Correlation between expression levels of PRLR and GnRH in the hypothalamus}

The relative expression levels of PRLR in the hypothalamus, pituitary, and ovaries or testis were positively 


\begin{tabular}{|c|c|}
\hline & GGGCACCAAGAGGAGCCCTGGASGTTTGCTGTGTTGARASAGARAGCARAGCCTCTTCGGG TCACTGCATATTCTTTGTTGACCACTT \\
\hline 31 & TCTCCGACTCCTCCCCCTTCTCAGGGASGG TG TGACTCASATCCCCGGAGCCCCCTCAAGGATTGTCAAGCTGCAGCTCAGTGCTGCTT \\
\hline 181 & GCTGGGASATARGGAASGAAATTTGACACCAAGCATCATCTTGGCTACTGAATATCATCTCTGAGGTCACTCGACTTAAAGGGCATTTT \\
\hline 11 & TTATTATTGTTTTGTCGAGTCTTGGTGGASGAGGG TCCCTTGATCATGCARACGGATCCAGGGGRRATCAGARATTTTCTTGTCCACCT \\
\hline 51 & ATTCTCTGCATTCCGACTCTCCCAACTACCTCCGTCACCTTGTARASTTGGCAGGCTCTGGACCTTTTGCATGCTGGAGASARTCACTGT \\
\hline 451 & TTTGCCTCCAGCARGABACATGCATGTTGCAACACTGACTCCTCCTCTAGTGAAGASGGGTGASCARGTGCACCGAGTTGAGCTTCTTC \\
\hline 11 & ACAGAGACACGCGGCTGGCGACTGCGGAGCAGAGGCTARASTCCCCAGACGCCGGGTCTTCTGGGCTGGGCTTTCCCCTCCTCCCTGTTC \\
\hline 31 & IGTCTTACTCGCTCTTCTGCGTTCTGGATTTTACCCAGGGCTCAGGAACAGCTTTACCTGCAGTGAGAAAGCTCGCGTCCTTTGCAGGA \\
\hline 21 & AGTACTCACCGCCTGGTATGGCAGACTTTTCTCCCCGGCASAACTASAGGACACTTCTCCTATCTGTGAAGGGAGCCTCTGATCCATTGC \\
\hline 311 & CTGTATCAAGAASCAGGGGCCAACATGAAGGAAGCATGCCATCTGCCCTTGCTTTCATCCTCCTTTTTCTCAMCATCAGGCTTCTTAAT \\
\hline & 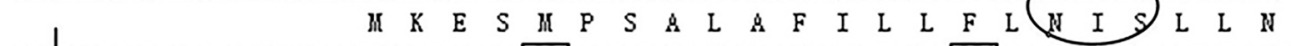 \\
\hline 301 & 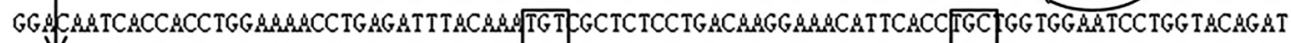 \\
\hline 23 & 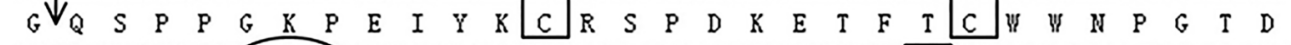 \\
\hline 391 & GGAGGACTTCCTACCATTACTCXCTGACTTACAGCAAAGAGGAGAGARARCCACCCATGASTGTCCAGACTACGARACCAGTGGCCCC \\
\hline 53 & 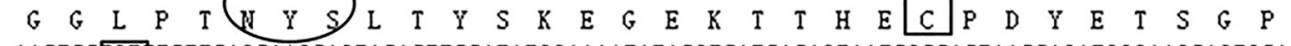 \\
\hline 1081 & AACTCC FGT TCTTCAGCARGCAGTACACTTCCATATGGARATATACGTCATCACAG TAATCGCCACTAACCAGATGGGAAGCAGTGCA \\
\hline 83 & $\begin{array}{llllllllllllllllllllllllllllll}N & S & C & F & F & S & K & Q & Y & T & S & I & W & K & I & Y & V & I & T & V & I & A & T & N & Q & M & G & S & S & A\end{array}$ \\
\hline 1171 & TCAGATCCACTTTATGTGGATG TGACTTACATAGTTGACCAGAGCCTCCTCGGARCCTGACTCTAGAAGTGAAACAGCTARAAGACASA \\
\hline 13 & $\begin{array}{lllllllllllllllllllllllllllllll}S & D & P & L & Y & V & D & V & T & Y & I & V & E & P & E & P & P & R & N & L & T & L & E & V & K & Q & L & K & D & K\end{array}$ \\
\hline 1261 & ASGACATATCTGTGGATARASTGGTCCCCACCCACCATARCTGATGTARARACTGGTTGGTTTACARTGGARTATGARATTCGATTGASG \\
\hline 143 & $\begin{array}{llllllllllllllllllllllllllll}Y & L & W & I & K & W & S & P & P & T & I & T & D & V & K & T & G & W & F & T & M & E & Y & E & I & R & L & K\end{array}$ \\
\hline 351 & 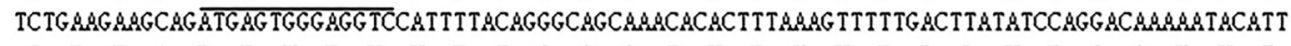 \\
\hline 13 & 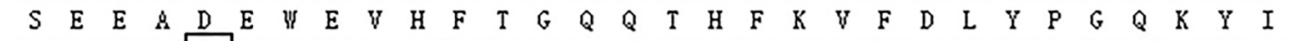 \\
\hline 1441 & GTCCAGACTCGCFGC ASGCCAGACCATGGATACTGGAGTAGATGGGCCABGAGRATTCCATTGARATACCCAATGACTTCACCTTGASA \\
\hline$\frac{203}{1531}$ & 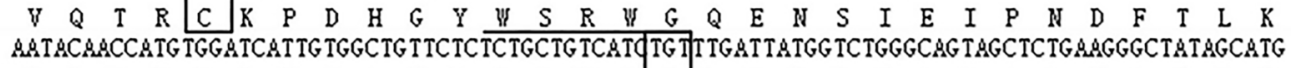 \\
\hline 33 & N I I M U I I V A V L S A V ILC L I M V W A V A L K G Y S M \\
\hline 21 & 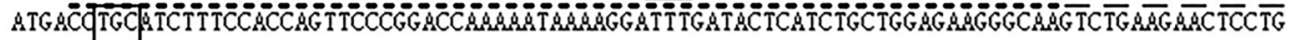 \\
\hline & $\begin{array}{l}M \\
M\end{array}$ \\
\hline & 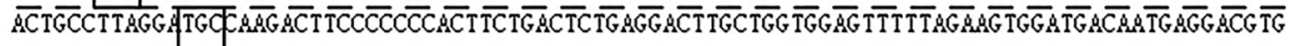 \\
\hline 93 & 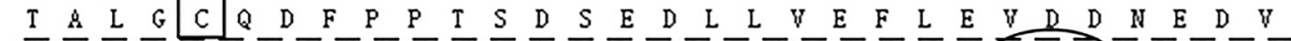 \\
\hline & 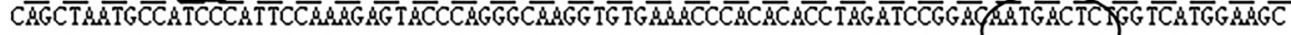 \\
\hline & 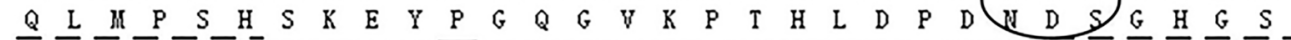 \\
\hline & 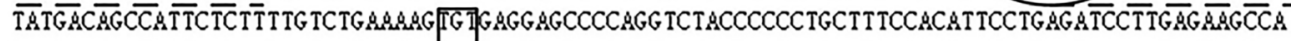 \\
\hline 53 & $\underline{\mathrm{Y}} \underline{\mathrm{D}} \underline{\mathrm{S}} \underline{\mathrm{H}} \underset{\mathrm{S}}{\mathrm{L}} \underline{\mathrm{L}}-\mathrm{S}$ \\
\hline & 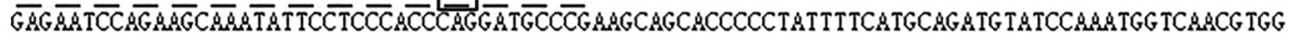 \\
\hline 183 & 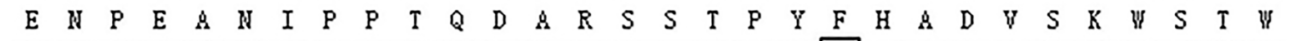 \\
\hline 2071 & CCTTTGCTGCCTAGCCAGCACATGACCAGATCTCCTTATCACAGCATTGCTGATGTG FGAAGCTAGCCGGAGG TCCTG TAGATACGCTC \\
\hline & 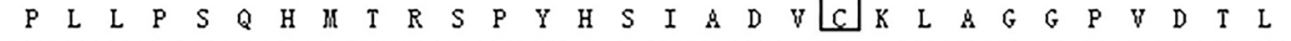 \\
\hline 1161 & 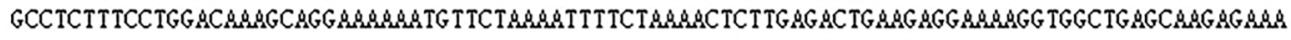 \\
\hline & $\begin{array}{lllllllllllllllllllllllllllllll}A & S & F & L & D & K & A & G & K & N & V & L & K & F & S & K & T & L & E & T & E & E & E & K & V & A & E & Q & E & K\end{array}$ \\
\hline & GARSAGCTTCCATTCTGAGACTARACASARCACATCATGGCCCCTGCTTCAGARASATGCCCCCATTGTCTACGCTARACCCCCAGAT \\
\hline & 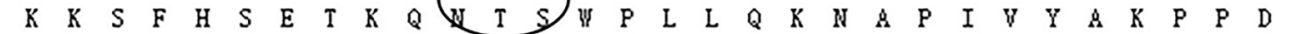 \\
\hline & TATGTGGAGATTCACASAGTCAACAASGATGGAGTATTATCATTGTTCCCCASACAGASGASASTGACCAGGTGGAGASGCCTGGGGTT \\
\hline & $\begin{array}{lllllllllllllllllllllllllllllll}Y & V & E & I & H & K & V & N & K & D & G & V & L & S & L & F & P & K & Q & K & E & N & D & Q & V & E & K & P & G & V\end{array}$ \\
\hline & CCAGARACCAGTAAGGAATATACCABGG TG TCTGGGGTTATGGATGACAACATCCTGGTGTTAGTGCCAGATTCACGAGCTGAGAACACA \\
\hline & $\begin{array}{lllllllllllllllllllllllllllllll}P & E & T & S & K & E & Y & T & K & V & S & G & V & M & D & D & N & I & L & V & L & V & P & D & S & R & A & E & N & T\end{array}$ \\
\hline & GCTTTGTTTGCGGASTCAGCCASGASGGTTCCACCATCGCTTGAACAGATCAGTCYCGASAGATCTGGCTAGCTTTGCTGCAACCTCC \\
\hline b. & $\begin{array}{llllllllllllllllllllllllllllll}A & \mathrm{~L} & \mathrm{~F} & \mathrm{~A} & \mathrm{E} & \mathrm{S} & \mathrm{A} & \mathrm{K} & \mathrm{K} & \mathrm{V} & \mathrm{P} & \mathrm{P} & \mathrm{S} & \mathrm{L} & \mathrm{E} & \mathrm{Q} & \mathrm{N} & \mathrm{Q} & \mathrm{S} & \mathrm{A} & \mathrm{K} & \mathrm{D} & \mathrm{L} & \mathrm{A} & \mathrm{S} & \mathrm{F} & \mathrm{A} & \mathrm{A} & \mathrm{I} & \mathrm{S}\end{array}$ \\
\hline & AACAATTGCAGACTCCAGCTGGGCAGGCTGGACTACCTGGATCCTACSTGCTCACACACTCCTTTCACTGATTGTTAGACTTATGGAAC \\
\hline 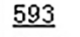 & 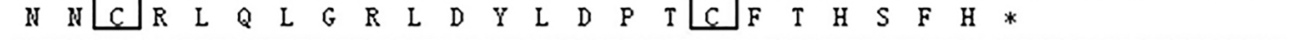 \\
\hline & \\
\hline & \\
\hline
\end{tabular}

Figure 1. Prolactin receptor (PRLR) cDNA sequence and inferred amino acid sequence of Cricetulus barabensis ( $n=6)$. Top sequence is the nucleotide sequence and bottom sequence indicates the deduced amino acid residues. The nucleotide sequence deduced to amino acid residues was open reading frame, including 1848-base-pairs (835-2682 bp). The nucleotide sequence 1-834 bp is in the 5'-untranslated region, and the nucleotide sequence 2683-2866 bp is in the $3^{\prime}$-untranslated region. The signal peptide (1-23 amino acid residues) is marked by an arrow, and the transmembrane domain (234-257 amino acid residues) is underlined by short dashed line. Two conserved amino acid residue sequences at the intracellular domain are underlined by long dashed lines. Two prolactin-binding motifs (WIKWS and WSRWG) at the extracellular domain are marked by solid line. The cysteines are boxed and the Asn-linked glycosylation sites are circled. 

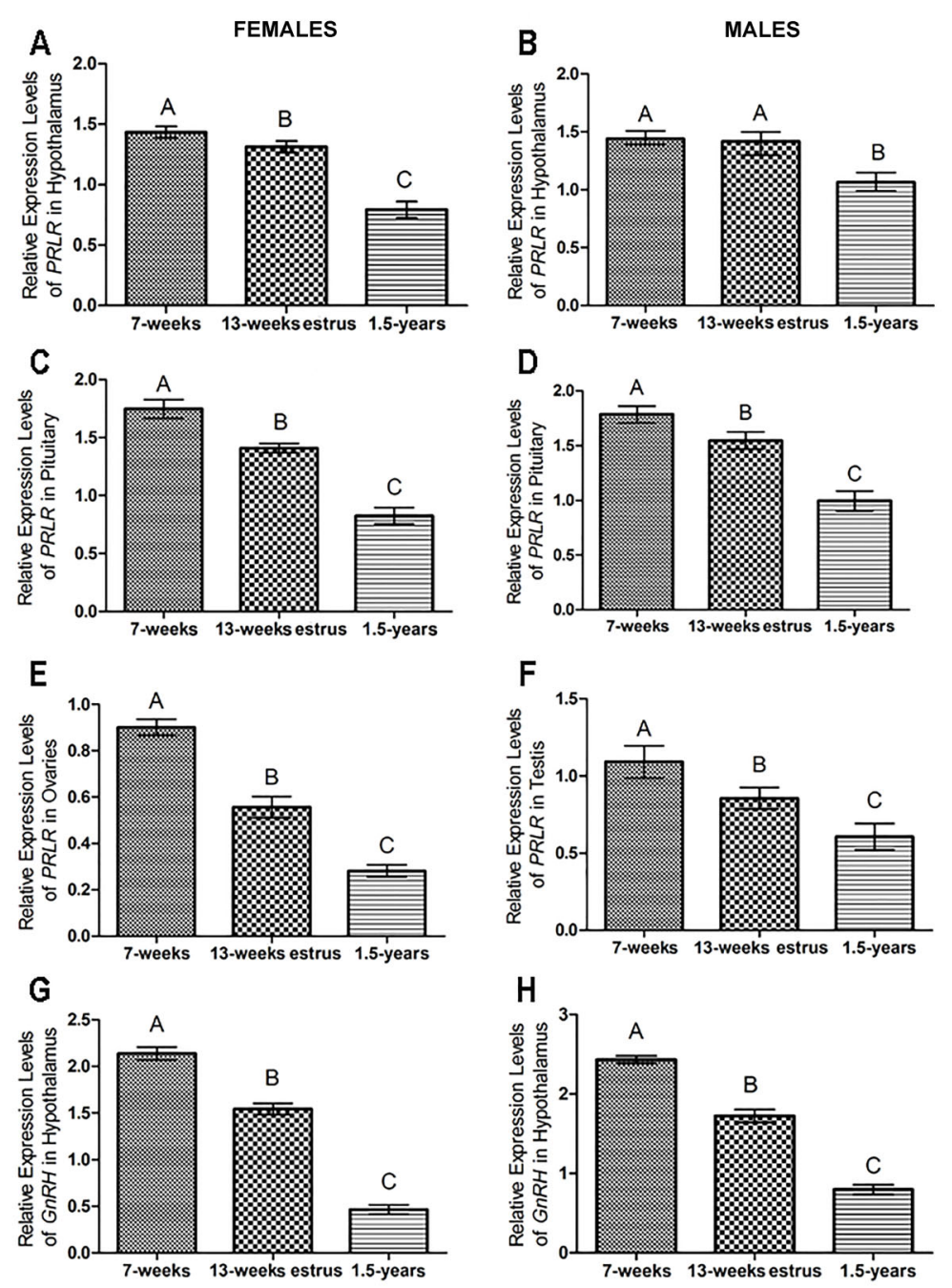

Figure 2. Relative expression levels of prolactin receptor (PRLR) mRNA in the hypothalamus, pituitary, and ovaries or testis, and GnRH mRNA in the hypothalamus of the striped hamster at different developmental stages ( 7 weeks, $n=6 ; 13$ weeks, $n=6,1.5$ years, $n=6)$ for $(\mathbf{A}, \mathbf{C}, \mathbf{E}, \mathbf{G})$ females and $(\mathbf{B}, \mathbf{D}, \mathbf{F}, \mathbf{H})$ males. The data are reported as means \pm SE. Different upper-case letters indicate significant differences $(P<0.01)$. (ANOVA).

correlated with the expression levels of $\mathrm{GnRH}$ in the hypothalamus of the striped hamster (Figure 5).

\section{Discussion}

In this study, we found that the PRLR mRNA levels in the hypothalamus, pituitary, ovaries or testis were developmental-stage-dependent with the highest level at sub-adult stage and lowest level at old stage. PRLR mRNA levels were highest in pituitary, medium level in hypothalamus, and lowest in ovaries or testis. PRLR mRNA was significantly higher in males than in females, except in the hypothalamus and pituitary from 7-week-old striped hamsters. Moreover, the PRLR mRNA levels in the hypothalamus, pituitary, and ovaries or testis were positively correlated with the expression levels of $\mathrm{GnRH}$ in the hypothalamus.

The high degree of identity between the striped hamster PRLR sequence and those of the golden hamster, Mus musculus, and Rattus norvegicus is illustrated in Supplementary Figure S1. The deduced signal peptide of PRLR in the striped hamster and the golden hamster contain 23 amino acid residues, while that of Mus musculus and Rattus norvegicus have 19 amino acid residues. Twelve cysteines were detected in the PRLR of the striped hamster, the golden hamster, and Mus musculus, but only eleven cysteines were detected in the Rattus norvegicus. That is because the nucleotide of Mus musculus PRLR at the ORF 1765 T was substituted by the nucleotide $C$, and the code TGC for cysteine was 

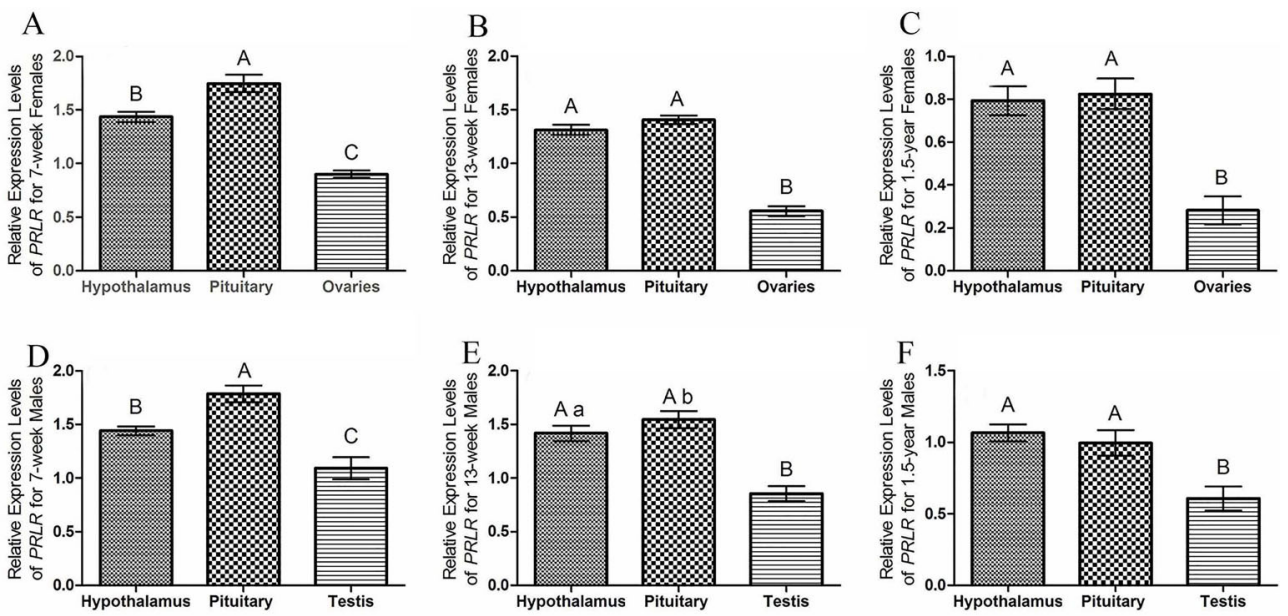

Figure 3. Relative expression levels of PRLR mRNA among the hypothalamus, pituitary, and ovaries or testis in the striped hamster at different developmental stages ( 7 weeks, $n=6 ; 13$ weeks, $n=6,1.5$ years, $n=6$ ). Relative PRLR mRNA of the hypothalamus, pituitary, and ovaries from (A) 7-week, (B) 13-week estrous, and (C) 1.5-year female striped hamsters. Relative PRLR mRNA of the hypothalamus, pituitary, and testis from (D) 7-week, (E) 13-week estrous, and (F) 1.5-year male striped hamsters. The data are reported as means $\pm S E$. Different lower-case letters $(P<0.05)$ and different upper-case letters $(P<0.01)$ indicate significant differences. (ANOVA).

A

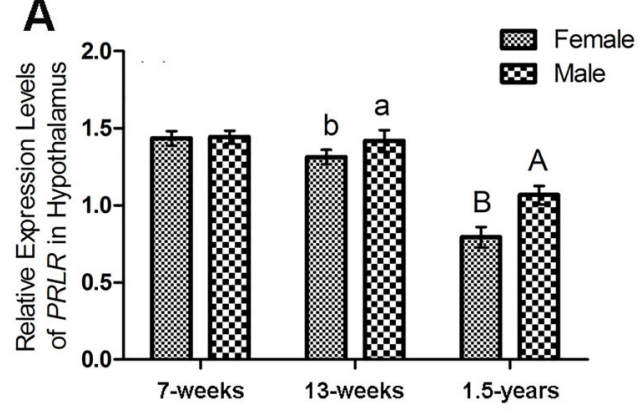

C

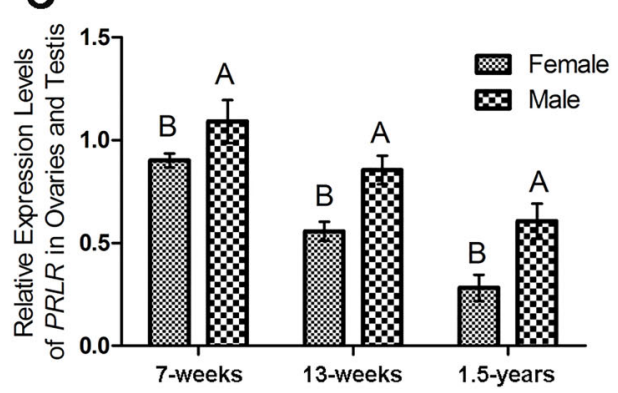

B
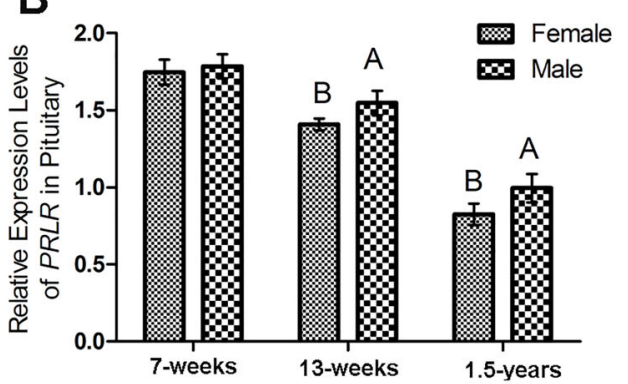

D

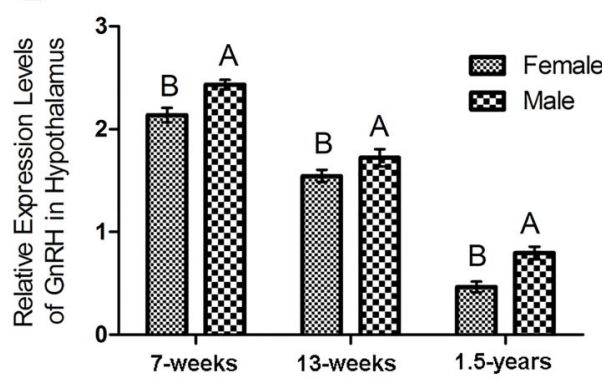

Figure 4. Relative expression levels of prolactin receptor (PRLR) mRNA between female and male striped hamsters $(7$ weeks, $n=6$; 13 weeks, $n=6,1.5$ years, $n=6$ ) in $(\mathbf{A})$ hypothalamus, $(\mathbf{B})$ pituitary, $(\mathbf{C})$ female ovaries and male testis, and (D) GnRH mRNA in the hypothalamus. The data are reported as means \pm SE. Different lower-case letters $(P<0.05)$ and different upper-case letters $(P<0.01)$ indicate significant differences (t-test).

transferred into CGC code for arginine. Five potential Asnlinked glycosylation sites were detected in the striped hamster and the golden hamster, while four sites were detected in the Mus musculus and Rattus norvegicus, as 1022 A was substituted by $G$, and the code AAT for asparagine was changed to AGT for serine. This nucleotide substitution may lead to some differences in regulating the structure and activity of PRLR. Two prolactinbinding motifs at the extracellular domain of PRLR were all found in the striped hamster, the golden hamster, Mus 

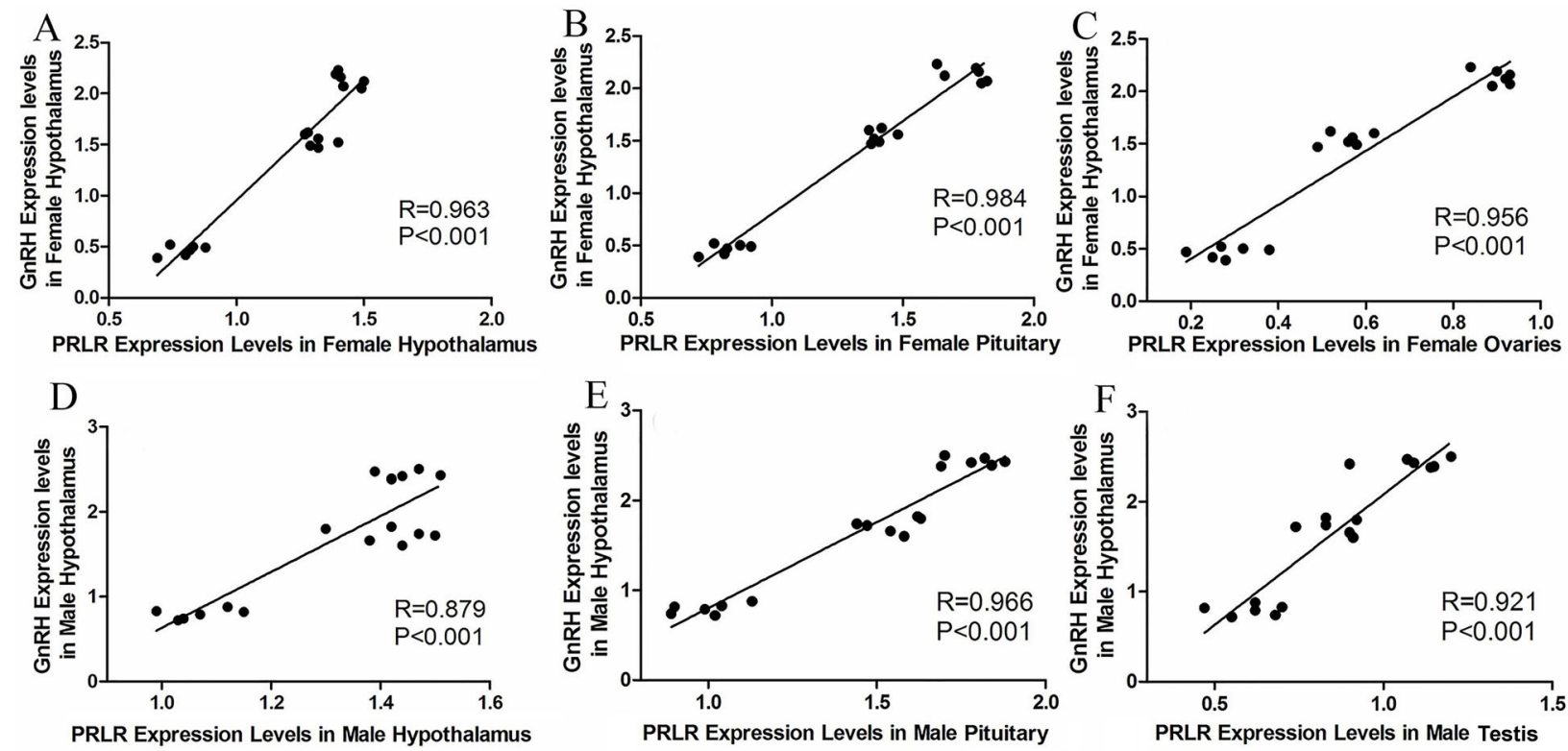

Figure 5. Correlations of the relative expression of prolactin receptor (PRLR) in the hypothalamus, pituitary, and ovaries (A-C) with that of $\mathrm{GnRH}$ in the hypothalamus of female striped hamsters. Correlations of PRLR mRNA in the hypothalamus, pituitary, and testis (D-F) with that of GnRH mRNA in the hypothalamus of male striped hamsters.

musculus, and Rattus norvegicus. The PRLR transmembrane region in the striped hamster also had high similarity with that of the golden hamster, Mus musculus, and Rattus norvegicus. The high similarity and some differences further demonstrated that the PRLR was conservative and divergent among various breeds. Two repeated units in the extracellular domain of the PRLR were also detected, which was similar to the chicken (24), the pigeon (25), and the turkey (12). The specific biological function of the repeated units remains to be identified, but each repeated unit contained four paired cysteine residues and a conserved ligand binding motif, which indicated that each PRLR may simultaneously interact with two PRL molecules.

The expression profile of PRLR in the striped hamster is developmental stage-dependent. $P R L R$ gene may play an important role in the attainment of sexual maturity (4), which is consistent with our results. In our study, the PRLR mRNA in the hypothalamus, pituitary, and ovaries or testis of the 7-week-old animals showed higher levels, which may promote the sexual maturity. PRLR may regulate the development of ovaries (3), and the transcription of PRLR in the ovaries is complicated and regulated by developmental stages and hormones (26). PRLR mRNA was also detected in the ovaries of the striped hamster, which indicated an important regulating factor for ovarian development.

The expression profile of PRLR in the striped hamster was tissue-dependent. PRLR was expressed in an extensive range of tissues, but the PRLR mRNA level varied among different tissues. The expression profile of PRLR in the striped hamster was also sex-dependent. A sex difference exists in pituitary and hypothalamic PRLR mRNA in Gallus domesticus, with lower levels in both tissues in females than that in males (27), which is consistent with our results in the pituitary and hypothalamus of 13-week-old and 1.5-year-old female and male striped hamsters. The PRLR mRNA in the pituitary and hypothalamus of 7-week-old striped hamsters had no significant sex difference.

Haplotypes of PRLR in chickens are significantly associated with egg production (4), which indicated an important role in reproduction; our results are consistent with this. The PRLR genotype is associated with the number of live sperm in boars (28) and the number of total offspring and live offspring in pigs (29). The secretion level of prolactin varies with the estrous cycle, and a secretion surge of prolactin occurs in the pre-ovulatory phase (30), which means that $P R L$ is an important regulating factor in ovulation.

In our study, significant correlations of PRLR mRNA in the hypothalamus, pituitary, and ovaries or testis with the expression levels of $\mathrm{GnRH}$ in the hypothalamus of the striped hamster showed that PRLR also had an important role in the reproduction of the striped hamster.

From our study, we concluded that the PRLR sequence in the striped hamster had a common and specific character compared with the PRLR sequence in other animals, and the expression profile of PRLR in the striped hamster was developmental-, sex- and tissue-dependent. PRLR plays 
an important role in gonad development and reproduction process, and its specific mechanism and effect need further study.

\section{Supplementary Material}

Click to view [pdf].

\section{References}

1. Mu CW, Wang YY, Ren WX. Studies on the biological characteristics and prevention and treatment for the striped hamster [in Chinese]. Gansu Agric Sci Technol 1999; 1: 39, CNKI: SUN: GSNK.0.1999-01-022.

2. Xue HL, Xu JH, Chen L, Xu LX. Genetic variation of the striped hamster (Cricetulus barabensis) and the impact of population density and environmental factors. Zoological Studies 2014; 53: 1-8, doi: 10.1186/s40555-014-0063-x.

3. Zhang X, Kang B, Zhang LN, Guo JR, Jiang DM, Ji H, et al. Gene expression profiles of $\mathrm{LH}$, prolactin and their receptors in female Zi geese (Anser cygnoides) during development. Folia Biol (Krakow) 2013; 61: 59-64, doi: 10.3409/fb61_ 1-2.59.

4. Zhang L, Li DY, Liu YP, Wang Y, Zhao XL, Zhu Q. Genetic effect of the prolactin receptor gene on egg production traits in chickens. Genet Mol Res 2012; 11: 4307-4315, doi: 10.4238/2012. October.2.1.

5. Freeman ME, Kanyicska B, Lerant A, Nagy G. Prolactin: structure, function, and regulation of secretion. Physiol Rev 2000; 80: 1523-1631, doi: 10.1152/physrev.2000.80.4.1523.

6. Ben-Jonathan N, LaPensee CR, LaPensee EW. What can we learn from rodents about prolactin in humans? Endocr Rev 2008; 29: 1-41, doi: 10.1210/er.2007-0017.

7. Howell-Skalla LA, Bunick D, Nelson RA, Bahr JM. Testicular recrudescence in the male black bear (Ursus americanus): changes in testicular luteinizing hormone-, follicle-stimulating hormone-, and prolactin-receptor ribonucleic acid abundance and dependency on prolactin. Biol Reprod 2000; 63: 440-447, doi: 10.1095/biolreprod63.2.440.

8. Higashimoto $\mathrm{Y}$, Nakao N, Ohkubo T, Tanaka M, Nakashima K. Structure and tissue distribution of prolactin receptor mRNA in Japanese flounder (Paralichtys olivaceus): conserved and preferential expression in osmoregulatory organs. Gen Comp Endocrinol 2001; 123: 170-179, doi: 10.1006/gcen.2001.7660.

9. Bole-Feysot C, Goffin V, Edery M, Binart N, Kelly PA. Prolactin (PRL) and its receptor: actions, signal transduction pathways and phenotypes observed in PRL receptor knockout mice. Endocr Rev 1998; 19: 225-268, doi: 10.1210/ edrv.19.3.0334.

10. Fiol DF, Sanmarti E, Sacchi R, Kültz D. A novel tilapia prolactin receptor is functionally distinct from its paralog. J Exp Biol 2009; 212: 2007-2015, doi: 10.1242/jeb.025601.

11. Leclerc B, Zadworny $D$, Bédécarrats $G$, Kuhnlein $U$. Development of a real-time (Q) PCR assay to measure variation in expression of prolactin receptor mRNA in the hypothalamus and pituitary gland during late embryogenesis in turkeys and chickens. Gen Comp Endocrinol 2007; 150: 319-325, doi: 10.1016/j.ygcen.2006.08.007.

\section{Acknowledgments}

This study was funded by the National Science Foundation of China (Nos. 31570377, 31670385, 31770455, and 31800308, and 31972283).

12. Zhang Y, Long Z, Li Y, Yi S, Shi Y, Ma X, et al. The second prolactin receptor in Nile tilapia (Oreochromis niloticus): molecular characterization, tissue distribution and gene expression. Fish Physiol Biochem 2010; 36: 283-295, doi: 10.1007/s10695-009-9355-1.

13. Boutin JM, Edery M, Shirota M, Jolicoeur C, Lesueur L, Ali S, et al. Identification of a cDNA encoding a long form of prolactin receptor in human hepatoma and breast cancer cells. Mol Endocrinol 1989; 3: 1455-1461, doi: 10.1210/ mend-3-9-1455.

14. Helm B, Schwabl I, Gwinner E. Circannual basis of geographically distinct bird schedules. J Exp Biol 2009; 212: 1259-1269, doi: 10.1242/jeb.025411.

15. VLi SG, Dai J, Zhang L, Zhang J, Zhang Z, Chen B. An association of elevated serum prolactin with phthalate exposure in adult men. Biomed Environ Sci 2011; 24: 3139, doi: 10.3967/0895-3988.2011.01.004.

16. Bonuccelli U, Murialdo G, Rossi G, Bonura ML, Polleri A, Murri L. Prolactin secretion in epileptic subjects treated with phenobarbital: sex differences and circadian periodicity. Epilepsia 1986; 27: 142-148, doi: 10.1111/j.1528-1157. 1986.tb03517.x.

17. Telleria CM, Parmer TG, Zhong L, Clarke DL, Albarracin CT, Duan WR, et al. The different forms of the prolactin receptor in the rat corpus luteum: developmental expression and hormonal regulation in pregnancy. Endocrinology 1997; 138: 4812-4820, doi: 10.1210/endo.138.11.5479.

18. Sugiyama $T$, Minoura $H$, Kawabe $N$, Tanaka M, Nakashima $\mathrm{K}$. Preferential expression of long form prolactin receptor mRNA in the rat brain during the oestrous cycle, pregnancy and lactation: hormones involved in its gene expression. $J$ Endocrinol 1994; 141: 325-333, doi: 10.1677/joe.0. 1410325.

19. Bridges RS, Hays LE. Steroid-induced alterations in mRNA expression of the long form of the prolactin receptor in the medial preoptic area of female rats: effects of exposure to a pregnancy-like regimen of progesterone and estradiol. Brain Res Mol Brain Res 2005; 140: 10-16, doi: 10.1016/ j.molbrainres.2005.06.011.

20. Li X, Su J, Lei Z, Zhao Y, Jin M, Fang R, et al. Gonadotropininhibitory hormone $(\mathrm{GnIH})$ and its receptor in the female pig: cDNA cloning, expression in tissues and expression pattern in the reproductive axis during the estrous cycle. Peptides 2012 ; 36: 176-185, doi: 10.1016/j.peptides.2012.05.008.

21. Huang DW, Di R, Wang JX, Chu MX, He JN, Cao GL, et al. Analysis on DNA sequence of goat RFRP gene and its possible association with average daily sunshine duration. Mol Biol Rep 2012; 39: 9167-9177, doi: 10.1007/s11033012-1789-3. 
22. Rutledge RG, Stewart D. A kinetic-based sigmoidal model for the polymerase chain reaction and its application to highcapacity absolute quantitative real-time PCR. BMC Biotechnology 2008; 8: 47, doi: 10.1186/1472-6750-8-47.

23. Livak KJ, Schmittgen TD. Analysis of relative gene expression data using real-time quantitative PCR and the 2 ( - delta delta C(T)) method. Methods 2001; 25: 402-408, doi: 10.1006/meth.2001.1262.

24. Chu MX, Zhang GX, Liu RZ, Wang JY, Fang L, Ye SC, et al. Cloning and sequence analysis of partial fragment of prolactin receptor gene in goats [in Chinese]. China Animal Husbandry \& Veterinary Medicine 2007; 34: 47-49.

25. Xing GD, Liu TZ, Liu QH, Fu ZH, Wang GL. Cloning and expression of goose prolactin receptor gene (gPRLR) [in Chinese]. J Nanjing Agric Univers 2007; 30: 97-101.

26. Centeno ML, Reddy AP, Smith LJ, Sanchez RL, Henderson JA, Salli NC, et al. Serotonin in microdialysate from the mediobasal hypothalamus increases after progesterone administration to estrogen primed macaques. Eur J Pharmacol 2007; 555: 67-75, doi: 10.1016/j.ejphar.2006.10.027.

27. Ohkubo T, Tanaka M, Nakashima K, Sharp PJ. Relationship between prolactin receptor mRNA in the anterior pituitary gland and hypothalamus and reproductive state in male and female bantams (Gallus domesticus). Gen Comp Endocrinol 1998; 111: 167-176, doi: 10.1006/gcen.1998.7099.

28. Kmiec M, Terman A. Associations between the prolactin receptor gene polymorphism and reproductive traits of boars. J Appl Genet 2006; 47: 139-141, doi: 10.1007/ BF03194613.

29. Serrano AB, Haro JGH, Hori-Oshima S, Espinosa AG. Prolactin receptor (Prir) gen polymorphism and associations with reproductive traits in pigs. J Anim Vet Adv 2008; 8: 469475.

30. Clevenger CV, Gadd SL, Zheng J. New mechanisms for PRLr action in breast cancer. Trends Endocrinol Metab 2009; 20: 223-239, doi: 10.1016/j.tem.2009.03.001. 